\title{
SIMPLIFIED AUTOMATIC WILSON CHAMBER
}

\author{
By L. F. Curtiss
}

ABSTRACT

A description of a simplified form of exhaust or vacuum type Wilson cloud chamber, built for demonstrating ray tracks, is given. No vacuum pump or quick-acting valve is required. By introducing some refinements this design seems adaptable also to the automatic photography of ray tracks.

\section{CONTENTS}

I. Introduction

II. Description of chamber.

\section{INTRODUCTION}

Of the many forms of the Wilson cloud chamber arranged for automatic operation, which have been devised, those operated on the exhaust, or "vacuum," principle of the original chamber built by C. T. R. Wilson, have proved most satisfactory. In this form the piston, forming the floor of the chamber, is pulled down by suddenly connecting the closed space beneath it with an evacuated chamber by means of a valve which can be opened instantaneously. The use of springs operating directly on the piston to produce the required motion does not give good results, since it is difficult to avoid a vibratory motion of the piston which interferes with the production of sharp tracks and may even cause the production of ghost tracks as the result of two rapidly succeeding expansions. Furthermore springs become troublesome to operate in the case of larger chambers where the tensions must be large to work against the back pressure of the atmosphere. Link motions and other direct mechanical drives which have been tried have failed to give sufficiently rapid expansion unless the piston is operated at the rate of 100 or more expansions per minute, which is entirely unsatisfactory for photographic purposes and leads to confusion of the observer where such apparatus is used to demonstrate ray tracks to those unfamiliar with their appearance. A further difficulty with rapidly successive expansions is that time is not allowed for temperature equilibrium to be restored, a requirement for sharp tracks.

The operation of chambers of the exhaust type requires an oil pump of fairly large capacity and a complicated valve mechanism. Whereas these items may not be serious disadvantages in the case of permanent laboratory equipment they make impractical the operation of small, more or less portable, chambers on this principle because of the bulk and cost of this accessory equipment. The writer has designed a small chamber of an exhaust type which eliminates the use of an oil pump or valve. It is therefore much more compact and portable than the usual form of chambers of this type. Likewise it is less expensive to build. These features make it particularly adaptable for the purpose of demonstrating ray tracks automatically. The 
following description is based on a small chamber recently built in this laboratory for demonstrating ray tracks and no attempt has been made to include those refinements which would be required to adapt this design for automatic photography of ray tracks. It seems quite possible, however, by using more care in the construction, and altering slightly the method of driving the auxiliary piston, to use this form of chamber for more precise work.

\section{DESCRIPTION OF CHAMBER}

The complete apparatus, shown diagramatically in Figure 1, consists of two parts - the cloud chamber proper, where the ray tracks are produced, and an auxiliary chamber or cylinder which serves alternately to produce the vacuum and as a valve to connect the evacuated space with the inclosed air space below the piston of the cloud chamber. The piston in the auxiliary cylinder is driven directly from an eccentric driven by an electric motor through a reduction gear, not shown, at about 30 revolutions per minute. This rate of

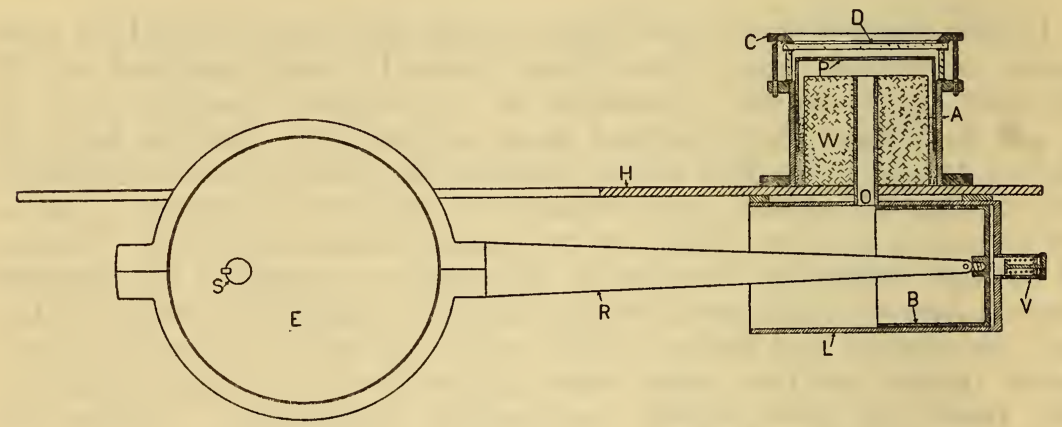

FIGURE 1.-Sectional diagram of simplified chamber

production of expansions is satisfactory for demonstration. To adapt this chamber for photographic work, however, a much slower rate would be necessary. This would entail more careful workmanship in making the auxiliary cylinder and a different type of mechanical drive for its piston.

The details of the apparatus are shown in section in Figure 1. The cloud chamber consists of a glass-walled cylindrical chamber closed on top by the glass disk, $D$, through which the ray tracks are observed. This disk is clamped down by screws through the metal ring, $C$, rubber gaskets being inserted in all joints to make them air-tight. The floor of the cloud chamber is formed by the "floating" piston, $P$, made of thin aluminum and working in an oil seal, as shown, within the brass cylinder, $A$. To reduce the volume of air which must be exhausted from the space below $P$, the usual practice of inserting a paraffined cylindrical wooden block, $W$, is followed. The auxiliary cylinder, $L$, is mounted horizontally beneath the base plate, $H$. It is provided with a close fitting brass piston, $B$, which is driven by the eccentric, $E$, mounted on the shaft, $S$. The eccentric and piston are connected by the rod, $R$. At the rate of operation indicated above, it is not necessary that the piston, $B$, be fitted to its cylinder, $L$, with great care since a fairly heavy lubricating oil may be used and a slight leak past this piston is not serious. 
A description of the cycle of operation will help to make clear the function of the various parts of the apparatus. Starting with the piston, $B$, in the position indicated in Figure 1, as the eccentric, $E$, rotates this piston will be drawn forward in its cylinder, $L$, producing a vacuum in the space behind the piston. At the instant the rear edge of the piston passes the orifice, $O$, this evacuated space is suddenly connected with the air space below the main piston, $P$, causing it to be drawn rapidly down against the wooden block, $W$. This produces the expansion in the cloud chamber required for the formation of ray tracks. As the eccentric continues to rotate, the piston, $B$, returns to its initial position which again opens the orifice, $O$, to atmospheric pressure, permitting the piston, $P$, to rise to its initial position ready for another expansion. As this cycle is repeated, air would accumulate

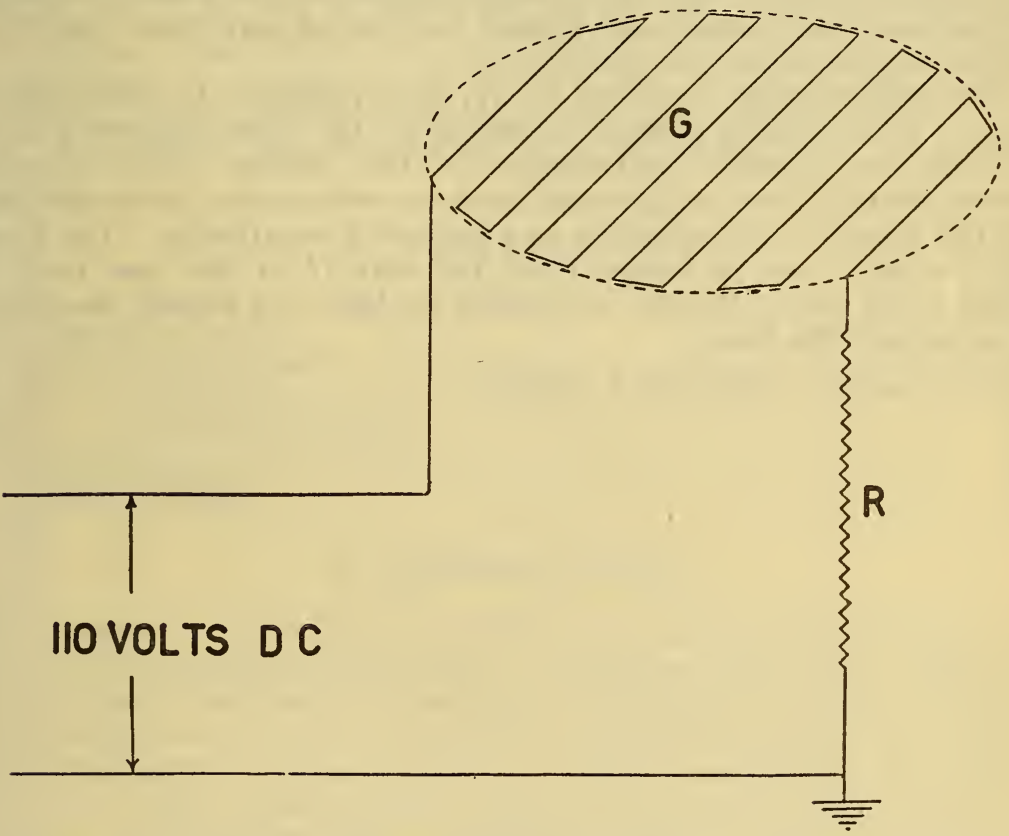

FIGURE 2.-Diagram showing electrical connections of grid for removing water vapor from under side of glass cover

behind the piston, $B$, building up a back pressure, which would cause the apparatus to fail to operate properly. To avoid this, a release valve, $V$, is provided to permit air to escape to the atmosphere on the return stroke of $B$. This valve is closed automatically by a spring on the working stroke as the piston, $B$, moves outward.

Some means of preventing the condensation of moisture on the underside of the glass disk, $D$, is required. This is ordinarily accomplished by water cooling the walls of the cylinder, $A$, so that the glass disk is at a slightly higher temperature than the piston, $P$. A more convenient method for a portable chamber is to mount a grid of fine wires spaced parallel to each other about $1 \mathrm{~cm}$ apart, connected in series. This grid, $G$, as shown in the diagram (fig. 2 ) is connected at one end with one side of the direct-current supply and at the other end through a high resistance to the opposite side of the direct-current 
supply. The high resistance, $R$, is so chosen that the temperature of the grid wires rises only slightly above that of the room. This grid may simultaneously be made to provide the electric field in the cloud chamber to sweep out old ions so that the general fog of the chamber is reduced, thereby increasing the sharpness of the new tracks on each expansion. To do this it is only necessary to take care to connect the grounded side of the direct-current supply to the free end of the high resistance which is then also connected to the base plate of the apparatus. Since nearly the whole potential drop occurs in the high resistance, the grid will then be at a potential above that of the piston, $P$, which is approximately equal to that of the supply, say 110 volts, which is quite sufficient. In using this arrangement for freeing the under side of the glass from moisture, it is desirable to have the voltage applied some time before observing the tracks, to permit temperatures to become steady and to drive off any water which has previously condensed on the glass.

The writer desires to thank B. W. Brown and L. L. Stockman for help in constructing a small chamber of the kind described above to test the practical advantages of the design. This chamber, approximately $7 \mathrm{~cm}$ in diameter, is of convenient size for demonstrating ray tracks with polonium as a source of $\alpha$-particles. The chamber is about $8 \mathrm{~cm}$ in height from the disk $D$ to the base plate $H$, which is $50 \mathrm{~cm}$ in length, supported on legs, not shown, to raise it $30 \mathrm{~cm}$ above the table.

Washington, February 3, 1932. 